\title{
On the boundary-layer control through momentum injection: Studies with applications*
}

\author{
V J MODI ${ }^{1}$ and T YOKOMIZO \\ ${ }^{1}$ Department of Mechanical Engineering, The University of British \\ Columbia, Vancouver BC, Canada V6T $1 Z 4$ \\ ${ }^{2}$ Department of Mechanical Engineering, Kanto Gakuin University, \\ Mutsuura, Kanazawa, Yokohama, Japan 236
}

\begin{abstract}
The concept of moving surface boundary-layer control, as applied to a Joukowsky airfoil, is investigated through a planned experimental programme complemented by numerical studies. The moving surface was provided by rotating cylinders located at the leading edge and/or trailing edge as well as top surface of the airfoil. Results suggest that the concept is quite promising, leading to a substantial increase in lift and a delay in stall. Depending on the performance desired, appropriate combinations of cylinder geometry, location and speed can be selected to obtain favourable results over a wide range of angle of attack. Next, effectiveness of the concept in reducing drag of bluff bodies such as a twodimensional flat plate at large angles of attack, rectangular prisms and three-dimensional models of trucks is assessed through an extensive wind tunnel test-programme. Results show that injection of momentum through moving surfaces, achieved here by introduction of bearing-mounted, motordriven, hollow cylinders, can significantly delay separation of the boundary-layer and reduce the pressure drag. The momentum injection procedure also proved effective in arresting wind-induced vortex resonance and galloping type of instabilities. A flow visualization study, conducted in a closed-circuit water tunnel using slit lighting and polyvinyl choride tracer particles, adds to the wind-tunnel and numerical investigations. It shows, rather dramatically, the effectiveness of the moving surface boundary-layer control (MSBC).
\end{abstract}

Keywords. Boundary-layer control; bluff body aerodynamics; drag reduction.

\section{Introduction}

Ever since the introduction of the boundary-layer concept by Prandtl, there has been a constant challenge faced by scientists and engineers to minimize its adverse effects

* The Sabita Chaudhury Memorial Lecture 
and control it to advantage. Methods such as suction, blowing, vortex generators, turbulence promotors etc. have been investigated at length and employed in practice with varying degrees of success. A vast body of literature accumulated over years has been reviewed rather effectively by several authors including Goldstein (1938), Lachmann (1961), Rosenhead (1966), Schlichting (1968), Chang (1970) and others. However, the use of moving wall for boundary-layer control has received relatively little attention. This is indeed surprising as the associate committee on aerodynamics, appointed by the National Research Council, specifically recommended more attention in this area (National Research Council 1966).

Irrespective of the method used, the main objective of a control procedure is to prevent, or at least delay, the separation of the boundary-layer from the surface. A moving surface attempts to accomplish this in two ways:. it prevents the initial growth of boundary-layers by minimizing relative motion between the surface and the free stream; and it injects momentum into the existing boundary-layer.

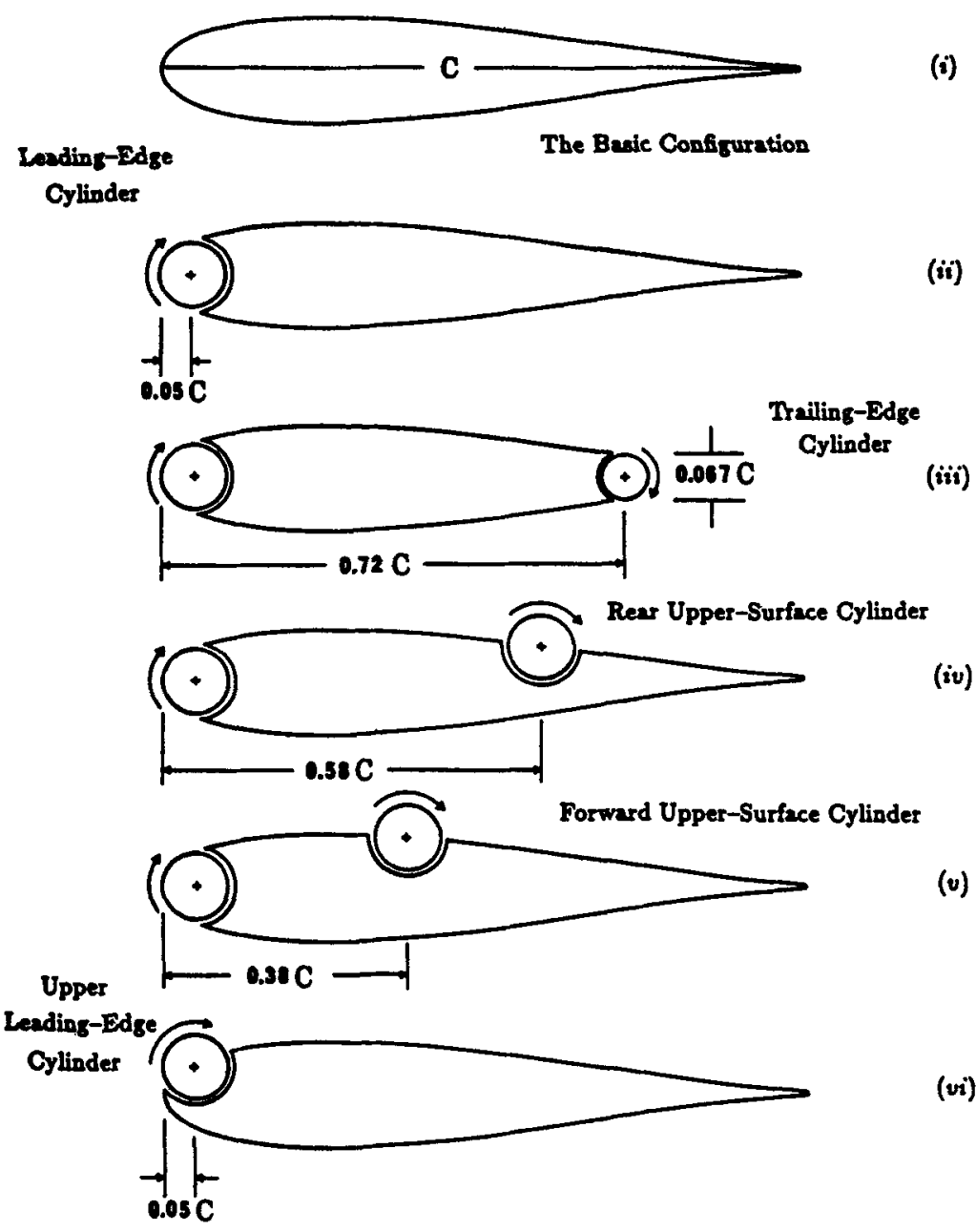

Figure 1. Various rotating-cylinder configurations studies with the Joukowsky airfoil model. 
Newton was probably the first one to observe the effect of moving wall boundary-layer control on the trajectory of a spinning ball (Thwaites 1960 , p. 215) without any appreciation as to the basis of the effect. Almost 200 years later Magnus (1853) studied lift generated by circulation and utilized the effect to construct a ship with a vertical rotating cylinder replacing the sail. Swanson (1961) and Iverson (1972) have presented excellent reviews of literature on the Magnus effect. As early as in 1910, Prandtl (Betz 1961) himself demonstrated his "ship of zero resistance" through flow around two counter-rotating cylinders, while Flettner (1925) applied the principle to ship propulsion in 1924 when he fitted large vertical rotating cylinders on the deck of the Buchau. A little later, in 1934, Goldstein (1938) illustrated the principle of boundarylayer control using a rotating cylinder at the leading edge of a flat plate. However, the most practical application of the moving wall for boundary-layer control was demonstrated by Favre (1938). Using an airfoil with the upper surface formed by a belt moving over two rollers, he was able to delay separation until the angle of attack $(\alpha)$ reached $55^{\circ}$ where the maximum lift coefficient of 3.5 was realized.

Efforts so far, though useful to an extent, were generally aimed at specific configurations and were scattered and lacked approach to the problem at a fundamental level in an organized fashion. From this point of view, the contribution by Modi and coworkers to the field is significant (Modi et al 1979-81, 1987a, 1987b, pp. 225-30, 1988, pp. 63-71, 1990a; Modi \& Mokhtarian 1985; Mokhtarian \& Modi 1984, pp. $167-75,1986$, pp. $322-30,1988$ ). They have studied in a comprehensive manner, the application of the moving surface boundary-layer control (MSBC) with reference to two-dimensional Joukowsky airfoils having one or more cylinders acting as momentum injecting elements (figure 1). The wind tunnel results were complemented by numerical as well as flow visualization studies (Modi 1991; Modi \& Yokomizo 1992, pp. 270-4). As can be expected the amount of information obtained is literally enormous. However, for conciseness, only some salient aspects of their investigations and typical results useful in establishing trends are recorded here.

\section{MSBC as applied to two-dimensional airfoils}

\subsection{Wind tunnel test-program}

The wind-tunnel model, a symmetrical Joukowsky airfoil of $15 \%$ maximum thickness to chord ratio, approximately $0.38 \mathrm{~m}$ along the chord and $0.68 \mathrm{~m}$ long, spanned the tunnel test-section, $0.91 \times 0.68 \times 2.6 \mathrm{~m}$, to create essentially a two-dimensional condition. The model was provided with pressure taps, suitably distributed over the circumference, to yield detailed information concerning the surface loading. It was supported by an aerolab six-component strain gauge balance and tested in a lowspeed, low-turbulence return-type wind tunnel where the airspeed can be varied from $1-50 \mathrm{~m} / \mathrm{s}$ with a turbulence level of less than $0.1 \%$. A Betz micromanometer with an accuracy of $0.2 \mathrm{~mm}$ of water was used to measure the pressure differential across the contraction section of $7: 1$ ratio. The rectangular test-section $(0.91 \times 0.68 \mathrm{~m})$ is provided with $45^{\circ}$ corner fillets that vary from $15.25 \times 15.25$ to $12 \times 12 \mathrm{~cm}$ to partly compensate for the boundary-layer growth. The spatial variation of velocity in the test-section is less than $0.25 \%$.

The rotating cylinders were supported by high-speed bearings housed in the brackets at either end of the model. They were driven by $1 / 4 \mathrm{hp}, 3.8 \mathrm{~A}$ variable speed 
motors, located outside the tunnel, through standard couplings. The configurations tested include the leading-edge, trailing-edge, forward upper-surface, rear uppersurface, upper-surface, and upper leading-edge cylinders. The model was provided with a total of 44 pressure taps, distributed over the circumference, to yield detailed information about the surface loading. However, once a section of the model was removed to accommodate a cylinder, the pressure taps in that section were lost. Although the pressure information over the small region represented by the uppersurface cylinder is not of particular significance, the corresponding data at the leading edge of the airfoil are crucial since it represents a high-suction region. Its measurement presented a challenging task. Locating pressure taps on the surface of the cylinder, typically rotating in the range of $2000-8000 \mathrm{rpm}$ offers considerable practical difficulty. The problem was resolved by measuring the pressure in the immediate vicinity of the cylinder rather than on the surface itself.

This was achieved in the case of the leading-edge cylinder by keeping the pressure taps stationary while the cylinder rotated. By locating the tap in a narrow ring, the width of which represented only a very small fraction of that of the cylinder, it was possible to ensure the continuity of flow over the entire surface and to obtain an estimate of the surrounding pressure. The leading-edge cylinder was provided with grooves to house the "pressure rings" which maintained the cylinder surface uniform.

The tests were conducted over an extended range of angles of attack and cylinder rotational speeds, corresponding to $U c / U=0,1,2,3,4$ at a Reynolds number of $4.62 \times 10^{4}$. Here $U c$ and $U$ correspond to the cylinder surface and free stream velocities, respectively. The choice of the Reynolds number in this case was dictated by vibration problems with multicylinder configurations operating at high rotational speeds (around $8000 \mathrm{rpm}$ for $U c / U=4$ ). The pressure plots were integrated in each case to obtain the lift coefficient. The lift was also measured independently using an aerolab six-component strain gauge balance to assess the two-dimensional character of the flow.

\subsection{Results and discussion}

The relatively large angles of attack used in the experiments result in a considerable blockage of the wind-tunnel test-section, from $21 \%$ at $\alpha=30^{\circ}$ to $30 \%$ at $\alpha=45^{\circ}$. The wall confinement leads to an increase in local wind speed at the location of the model, thus resulting in an increase in aerodynamic forces. Several approximate correction procedures have been reported in the literature to account for this effect. However, these procedures are mostly applicable to streamlined bodies with attached flow. A satisfactory procedure applicable to a bluff body offering a large blockage in a flow with separating shear layers is still not available.

With rotation of the cylinder(s), the problem is further complicated. As shown by the pressure data and confirmed by the flow visualization, the unsteady flow can be separating and reattaching over a large portion of the top surface. In the absence of any reliable procedure to account for wall confinement effects in the present situation, the results are purposely presented in uncorrected form.

2.2a Base airfoil: The pressure distribution data for the "base airfoil" (in absence of the modifications imposed by the leading-edge or upper-surface cylinder) are presented in figure 2 ., The leading edge was now formed by a snugly fitting plug (the nose fill-in section). Due to practical difficulties in locating pressure taps in the cusp 


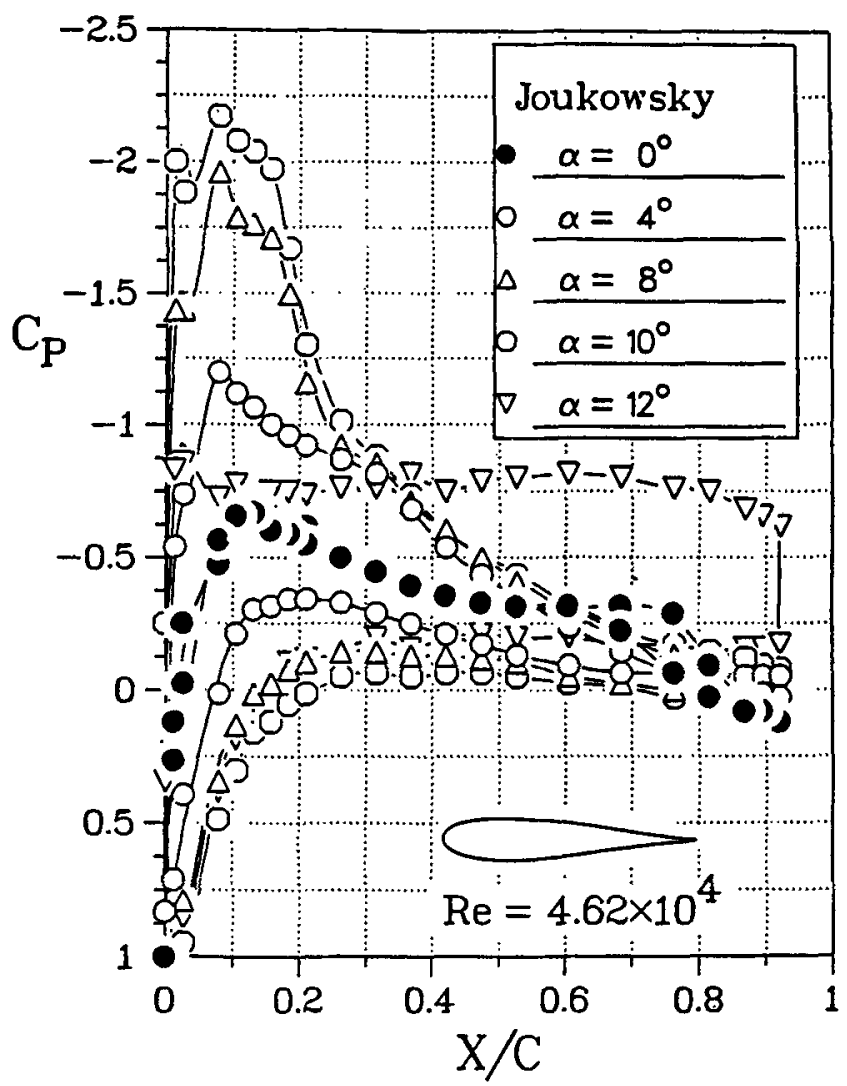

Figure 2. Experimentally obtained pressure distributions for the basic Joukowsky model.

region, there is an apparent discontinuity in the pressure plots near the trailing edge. However, this region has little importance in the present discussion. It is apparent that the airfoil, in absence of any modifications to its nose geometry, stalls at an angle of attack of around $10-12^{\circ}$. These results serve as reference to assess the effect of rotating cylinders in different locations.

Note that the wall confinement effect at $\alpha=10^{\circ}$ is relatively small, as the blockage ratio is around 7\%. More importantly, focus here is on the effect of the momentum injection due to the cylinder rotation with the airfoil at a given angle of attack. Results of the flow visualization study, presented later, emphasize this point.

2.2b Leading edge cylinder: Figure 3 summarizes the effects of modification of the airfoil with the leading-edge cylinder and the cylinder rotation. The base airfoil has a maximum lift coefficient of about 0.87 at an angle of attack of $10^{\circ}$. There is a penalty associated with the modified nose geometry as well as due to the gap, but even at the lowest rate of rotation of the cylinder $(U c / U=1)$ the lift and stall characteristics are significantly improved. The airfoil exhibits a desirable flattening of the lift curve at stall. The maximum lift coefficient measured with $U c / U=4$ was around 2 at $\alpha=28^{\circ}$, which is almost three times the lift coefficient of the base airfoil.

Typical pressure plots at a relatively larger angle of attack are presented in figure 4 to assist in more careful examination of the local flowfield. As the angle of attack of the airfoil is increased, the flow starts to separate from the upper surface close to the 

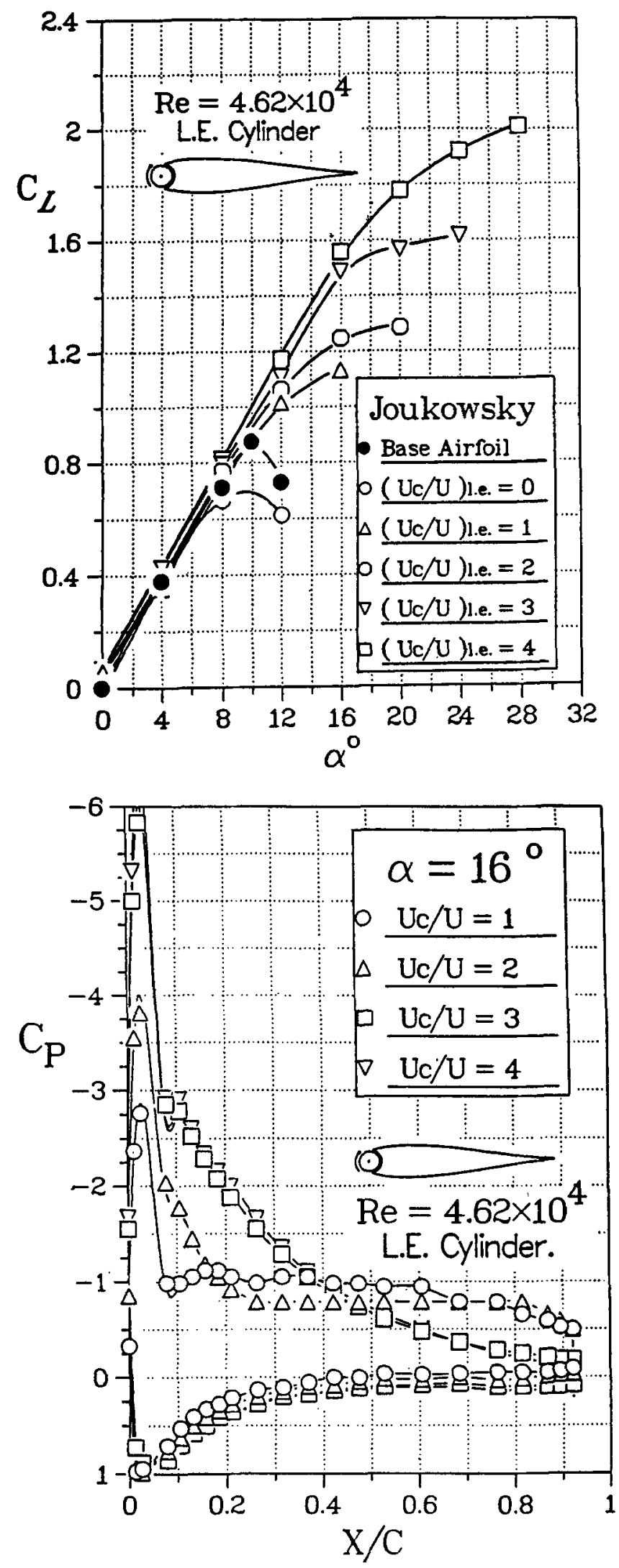

Figure 3. Effect of the leadingedge cylinder rotation on the lift and stall characteristics of the Joukowsky model.
Figure 4. Effect of increasing the rate of cylinder rotation on pressure distribution around the model at a relatively larger angle of attack of $\alpha=16^{\circ}$ 
leading edge. At $\alpha=16^{\circ}$, for example, the cylinder rotating at $U c / U=1$ only keeps the flow attached at the leading-edge. However, as the rate of rotation is increased, the size of the separated region is reduced, and at the higher rates of rotation the flow is again completely attached. Note that the point of separation on the upper surface clearly moves downstream with an increase in rate of rotation. The flow separates at around $X / C=25 \%$ with $U c / U=2$, near $X / C=80 \%$ when $U c / U$ is increased to three, and at the trailing edge with the highest $U c / U$ used. The flow visualization study discussed later substantiated this general behaviour rather dramatically.

2.2c Combined leading and trailing edge cylinders: The use of a leading-edge cylinder extends the lift curve, thus substantially increasing the maximum lift coefficient and delaying stall. On the other hand, the trailing-edge cylinder rotation results in an improvement in the lift coefficient, at a given angle of attack, before stall. In order to combine these effects, the base configuration was modified to include both the leading and trailing-edge cylinders. This phase of the test-programme examined the effect of individual and combined cylinder rotations. However, it is the combined effect of both the cylinders that is of interest here. Results shown in figure 5 suggest some benefit due to rotation of the two cylinders together. Although the increase in the $C_{L \text {, } \max }$ is rather modest (from 2.0 to $2 \cdot 5$, around $30 \%$ ) compared to the leadingedge cylinder case (sharp trailing edge, figure 3 ), the lift coefficient at a given $\alpha$ is indeed increased significantly, as expected, due to the leftward shift of the plots. As

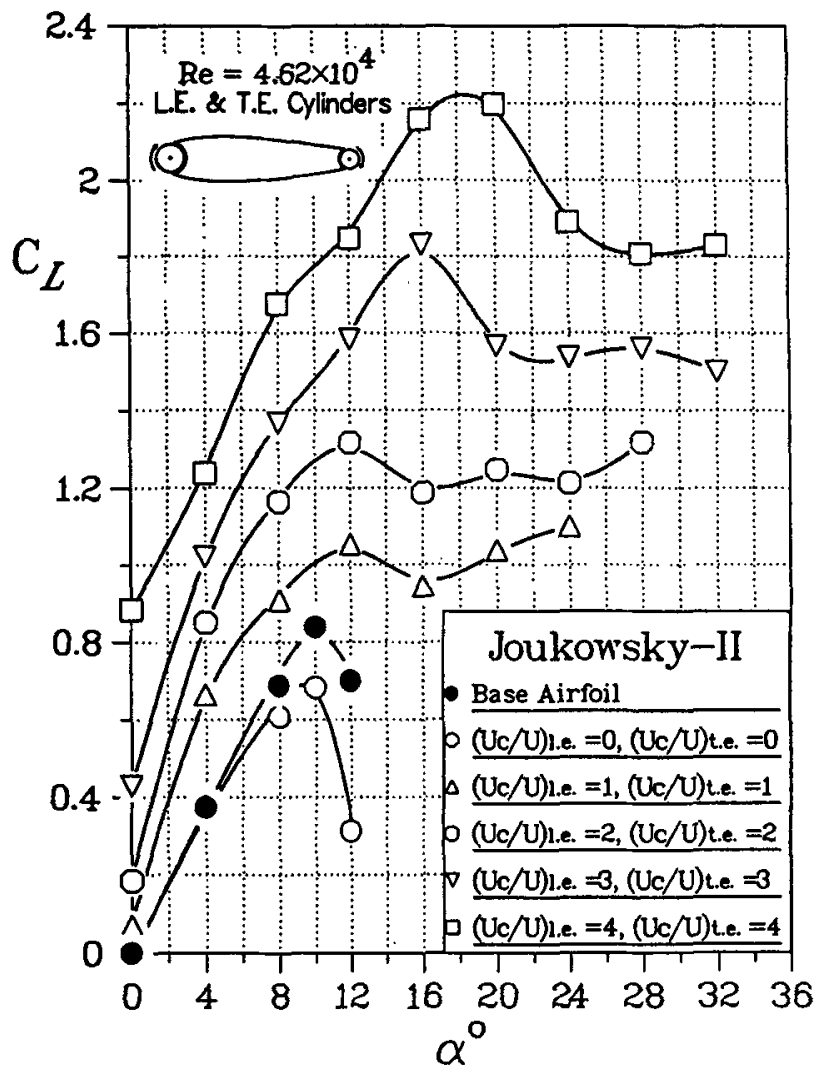

Figure 5. Variation of $C_{L}$ vs $\alpha$ for a modified Joukowsky airfoil with leading and trailing-edge cylinders. 
noted before, this is due to the added eirculation by the trailing-edge cylinder. For example, $C_{L}=0.8$ at $\alpha=8^{\circ}$ and $(U c / U)$ l.e. $=3$ (figure 3), whereas for the same angle of attuck and $(U c / U)$ l.e. $=(U c / U)$ t.e. $=3$ the corresponding $C_{L} \approx 1.57$, an increase of around $96 \%$. Similarly, $C_{L}=1.55$ for $\alpha=16^{\circ}$ and $(U c / U)$ l.e. $=4$. On the other hand, with both the cylinders rotating at $U c / U=4$, the lift coefficient is around $2 \cdot 48$, a further gain of about $60 \%$. Note, the maximum lift coefficient attained with rotation of both the cylinders represents an increase of $195 \%$ with respect to the reference configuration ( $C_{L, \max }$ of about 2.6 vs 0.88 , figure 5 ).

2.2d Forward and rear upper-surface cylinders: The forward and rear upper-surface cylinders, located at 38 and $58 \%$ chord, respectively, were considered independently and with either operating in conjunction with the leading-edge cylinder. As can be expected, in the absence of rotation, their protrusion into the upper-surface flow had an adverse effect on the aerodynamic charac.uristics of the model. The flow separated at the location of the cylinder, resulting in lower lift and increased drag. On the other hand, with rotation, either of the upper-surface cylinders was successful in attaining a higher $C_{L, \max }$ and delaying the stall. In this respect, the forward upper-surface cylinder was particularly effective.

2.2e Upper leading-edge cylinder: Effectiveness of the combination of leading-edge and forward upper-surface cylinders suggested the possibility of replacing the two by a single rotating element. This avoids the practical complications associated with construction, installation and operation of two rotating cylinders.

The configuration, with a cylinder located at approximately $5 \%$ of the chord, was tested at cylinder speeds in the range of $U c / U$ up to 4 . The results are presented in figure 6. Compared to the leading edge cylinder study (figure 3), where for $U c / U=4$,

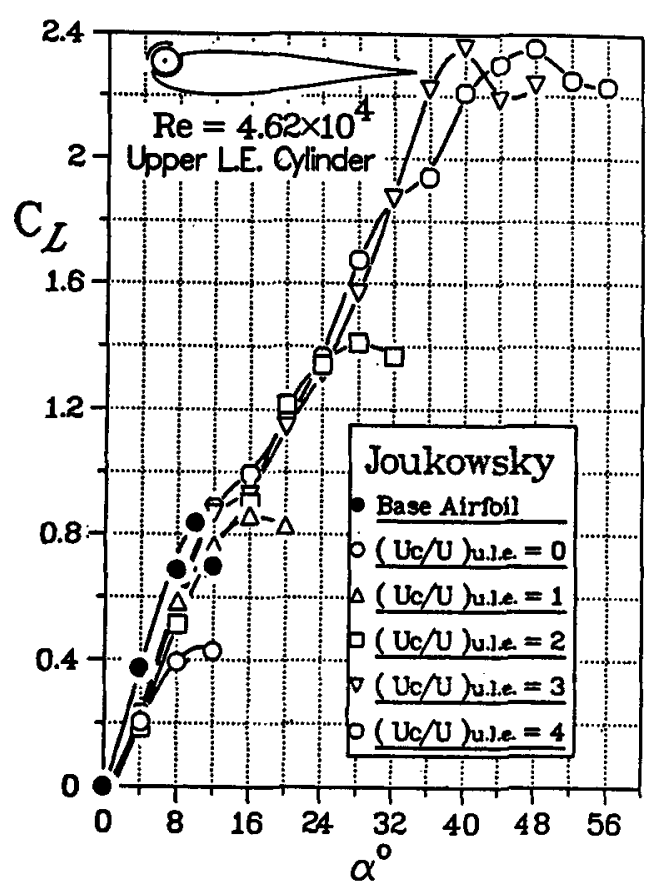

Figure 6. Lift and stall characteristics of the Joukowsky model as affected by the upper leading-edge cylinder rotation. 
$C_{L, \max } \approx 2$ and $\alpha_{\text {stall }} \approx 28^{\circ}$, now we have $C_{L, \max }=2.35$ with $\alpha_{\text {stall }} \approx 48^{\circ}$. This clearly suggests that location of the cylinder near the leading edge can significantly affect the airfoil performance. Thus, there is room for a systematic study to arrive at an optimum location. Even compared to the results obtained using the leading-edge cylinder together with the forward upper-surface cylinder, performance of the present single cylinder configuration appears attractive. Although the $C_{L, \max }$ is slightly lower (down from 2.73 to 2.35 ), the stall is delayed from around $40^{\circ}-48^{\circ}$. However, the main advantage would be the mechanical simplicity of working with one cylinder.

2.2f Comparative performance: With the vast amount of data obtained through a planned experimental program using the configurations presented earlier, it would now be useful to compare their distinctive features to help establish relative merits. Figure 7 attempts to achieve this objective. Results of the standard Joukowsky airfoil (symmetrical, $15 \%$ thickness), with its $C_{L, \max }=0.88$ and $\alpha_{\text {stall }}=10^{\circ}$, serve as reference for all the cases presented.

The leading-edge cylinder is quite effective in extending the lift curve, without significantly changing its slope, thus substantially increasing the maximum lift coefficient $(\approx 2)$ and delaying the stall angle $\left(28^{\circ}\right)$. Further improvements in the maximum lift coefficient and stall angle are possible when the leading-edge cylinder is used in conjunction with an upper-surface cylinder. This configuration also results in lower drag due to large recovery of pressure near the trailing edge, at moderately high angles of attack. The $C_{L, \max }$ realized with the leading-edge and forward uppersurface cylinders, was about $2.73\left(\alpha=36^{\circ}\right)$, approximately three times that of the base configuration.

A rotating cylinder on the upper side of the leading edge also proves to be very effective. Although the maximum coefficient of lift realized with its rotation is slightly

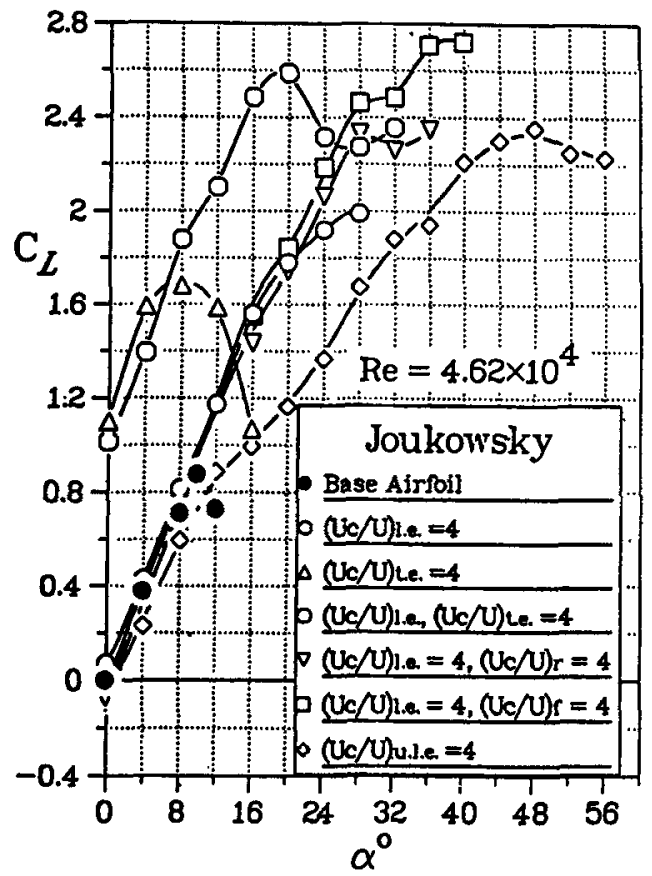

Figure 7. Plots to assess relative influence of different configurations studied on the lift and stall characteristics. 
lower $(\approx 2 \cdot 35)$, it does have a major advantage in terms of mechanical simplicity. Note that now the lift curve has a lower slope and is not an extension of the base airfoil lift curve. Hence, the lift at a given $\alpha$ is relatively lower; however, the stall is delayed to around $48^{\circ}$.

On the other hand, to improve the lift over the range of low to medium angles of attack $\left(\alpha \leqslant 20^{\circ}\right)$, the trailing-edge cylinder proves much more effective, particularly in conjunction with the leading-edge cylinder. The suction over the airfoil upper surface as well as the compression on the lower surface are increased dramatically with the higher rates of rotation of this cylinder, resulting in a substantial increase in lift $(\approx 195 \%)$.

Thus, depending on the intended objective in terms of desired $C_{L, \max }$ and stall angle, one can select an appropriate configuration to initiate a preliminary design.

\subsection{Flow visualization}

The flow visualization study was carried out in a closed-circuit water channel facility. The model was constructed from Plexiglas and fitted with a leading-edge cylinder, driven by a compressed-air motor. A suspension of fine polyvinyl chloride powder was used in conjunction with slit lighting to visualize streaklines. Both angle of attack and cylinder speeds were systematically changed and still photographs as well as a video movie were taken. The study showed, rather dramatically, the effectiveness of this form of boundary-layer control (figure 8). With the model at $\alpha=20^{\circ}$, and in absence of the cylinder rotation, a well-defined early separation resulting in a wide wake is quite apparent, with large-scale vortices sweeping away downstream. However, with the cylinder rotating at $U c / U=4$, an essentially attached flow is established over most of the upper surface of the airfoil.

At relatively lower rates of cylinder rotation, the flow character was found to be similar to that observed at $U c / U=1$, with the separation and reattachment regions progressively shifting downstream as the rotation rate increased. This is apparent through a progressive increase in $U c / U$ from 0 to 4 . In fact, the flow pattern was found to be quite unsteady with the vortex layer separating and forming a bubble on reattachment, the whole structure drifting downstream, diffusing, and regrouping at different scales of vortices. Ultimately the flow sheds large as well as small vortices. This unsteady character of the separating shear layer and the wake is clearly evident in the video. Thus the flow character indicated by the experimentally obtained timeaverage pressure plots appears to be a fair description of the process. Furthermore, this also suggests that analytical or numerical modelling of such highly complex and transient flow would pose a challenging problem.

\subsection{Numerical approaches}

The complex problem of multi-element airfoil with momentum injection was studied using two distinctly different numerical approaches:

(a) surface singularity distribution with boundary-layer correction;

(b) finite element integration of the Navier-Stokes equations.

2.4a Surface singularity approach: This numerical procedure is based on the surface singularity method described in detail by Mokhtarian (1988) in his doctoral dissertation. It accounts for the wall confinement and involves replacement of the 

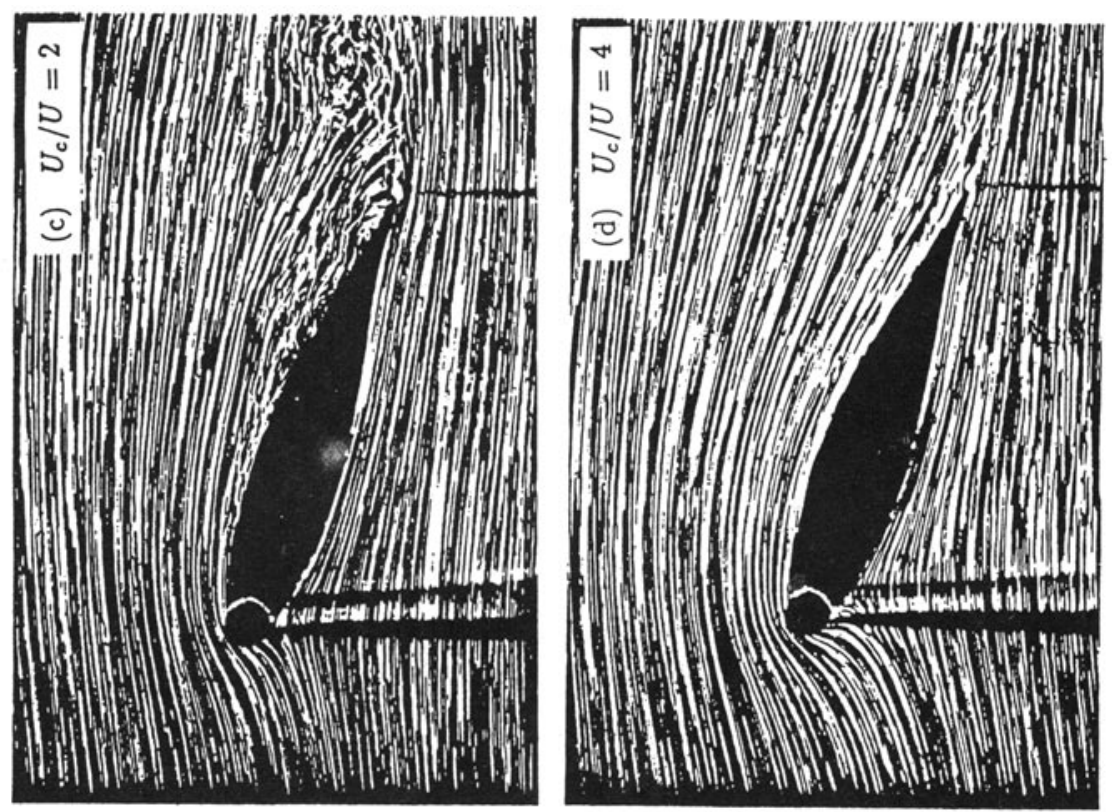

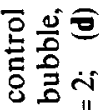

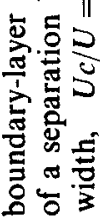

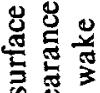

象育焉

을.

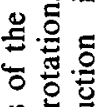

岁宫

尝章

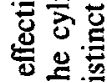

능

刍

荧荧
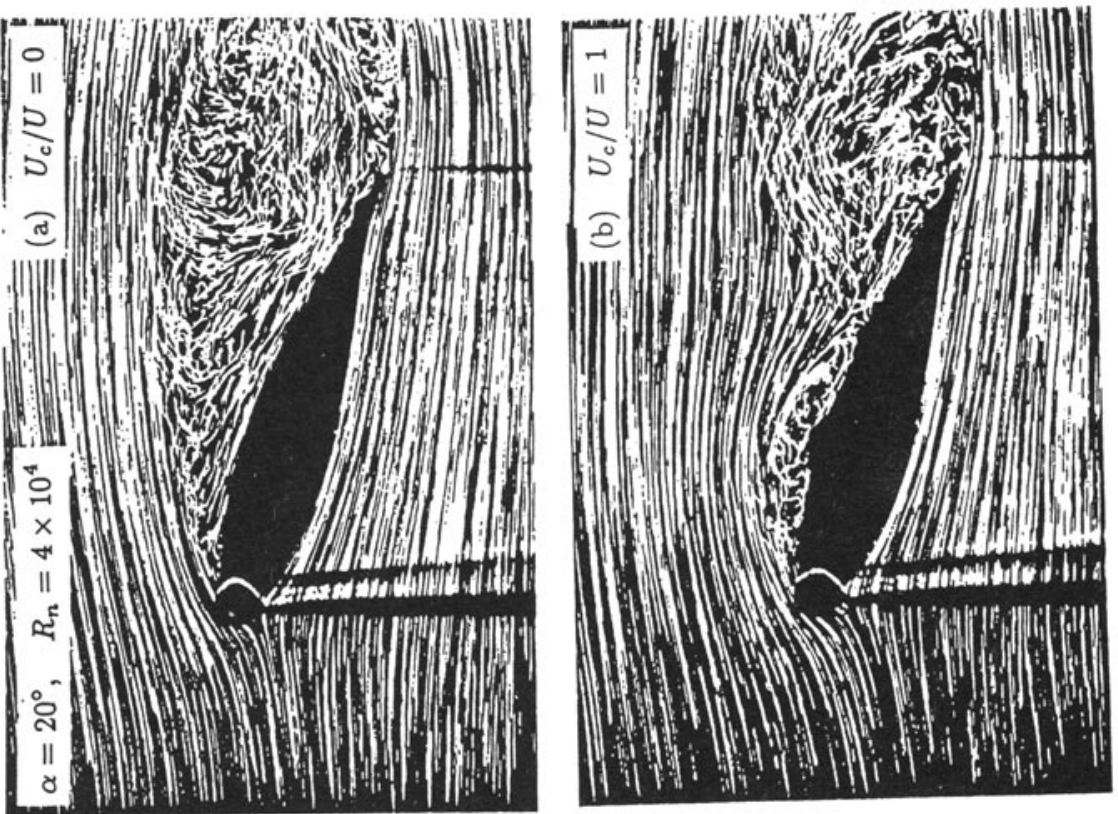

․․

害完

言

के

紊范

然品

的

등

苞产药

든은

帚焉

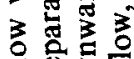

总要

흉 궁용

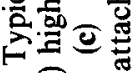

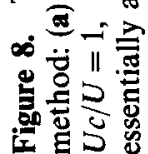


aerofoil and wind tunnel walls with vorticity distribution $\gamma$ in conjunction with appropriate constraint relations. Inclusion of a source within the contour of the airfoil models the wake when there is flow separation from the surface. A finite difference boundary-layer scheme is used to introduce viscous corrections. The scheme employs potential flow pressure distribution results to calculate the boundary-layer characteristics at the top and bottom surfaces starting from the stagnation point until the point of separation.

The procedure uses the displacement thickness to construct an equivalent airfoil and then iterates between the potential flow and boundary-layer scheme to converge to the final pressure distribution. Thus the objective is to match the outer potential flow solution with the inner boundary-layer prediction. The thin shear layer approximations of the Navier-Stokes equations for steady, two-dimensional, incompressible flow are used. The finite difference method employed for viscous correction is due to Keller \& Cebeci (1972). The eddy viscosity term is expressed as suggested by Cebeci \& Smith (1974) who treat the turbulent boundary-layer as a composite layer consisting of inner and outer regions with separate expressions for eddy viscosity in each region. The details of the formulation and the finite difference procedure followed are those given by Cebeci \& Bradshaw (1977).

Typical results for the Joukowski airfoil with upper leading edge cylinder are presented in figure 9. Wind tunnel test results are also included to facilitate comparison. Considering the complex character of the flow, the correlation is indeed excellent and the results can be used with confidence.

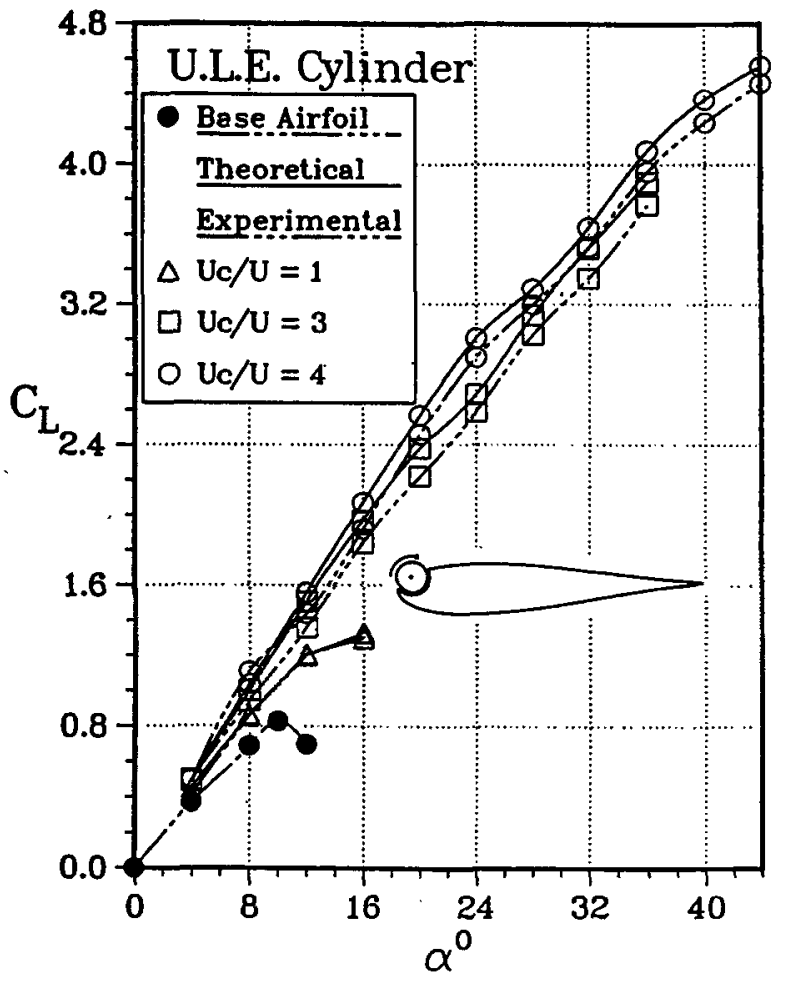

Figure 9. Variation of lift with angle of attack in presence of the MSBC as predicted by numerical and experimental procedures. Note that in spite of the complex character of the flow the correlation is excellent. 


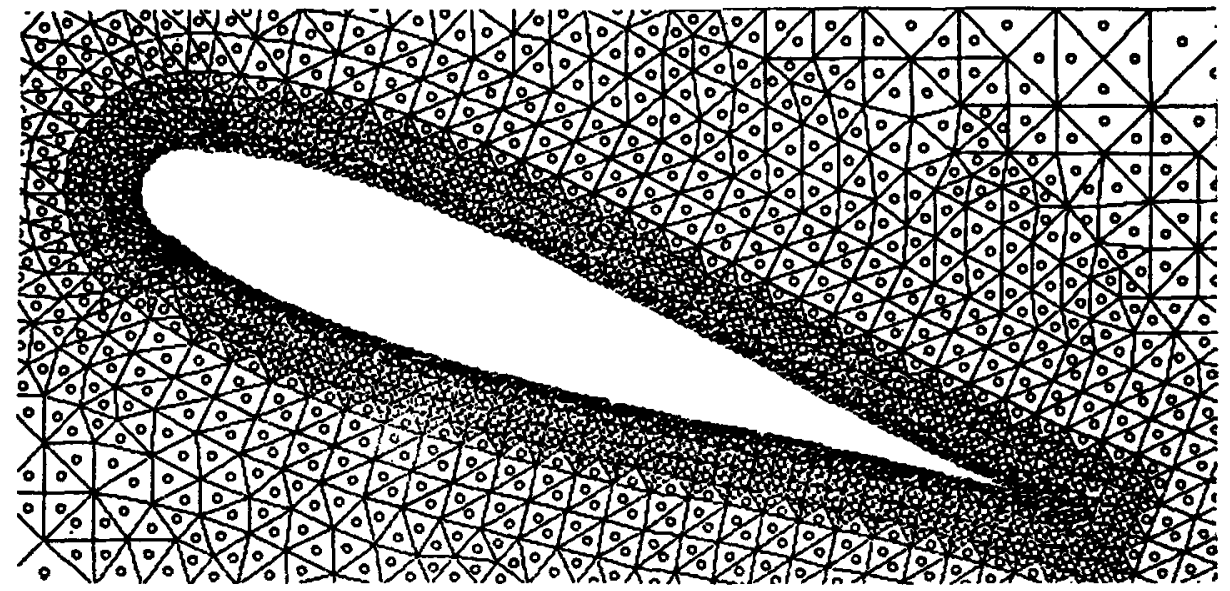

Figure 10. Triangular elements with spatially varying grid size used in the numerical simulation.

2.4b Finite element Navier-Stokes solution: Here the stream function-vorticity form of the Navier-Stokes equations are used in conjunction with the variable grid-size $(\approx 3000$ nodes, figure 10 ) finite element analysis (Modi \& Yokomizo 1992, pp. 270-4). Such a numerical solution of the two- and three-element airfoils with momentum
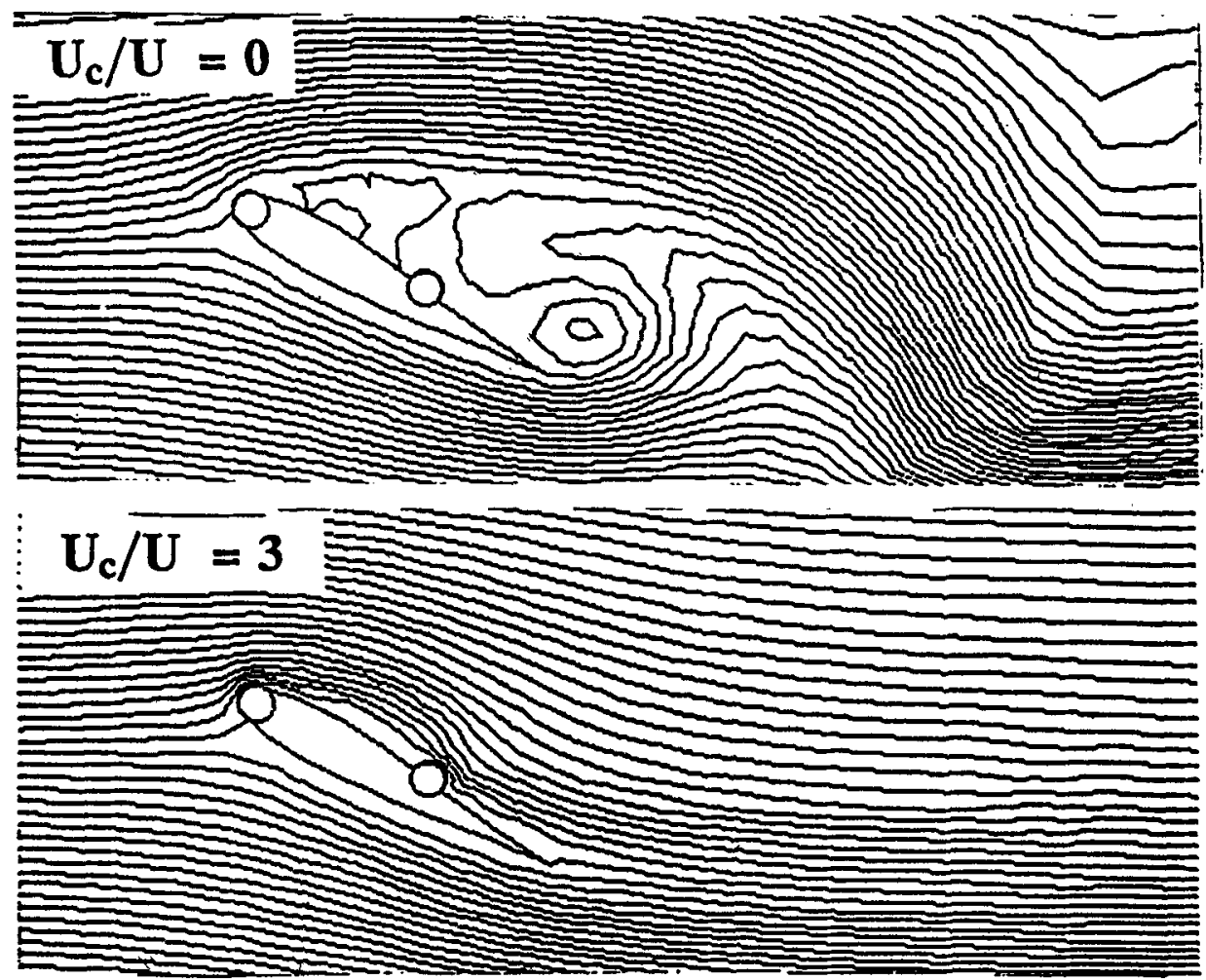

Figure 11. Typical numerically obtained flow patterns, for an airfoil at $\alpha=30^{\circ}$, showing effectiveness of the MSBC. 
injection has not been reported in literature. The parametric analysis involving a systematic variation of the speed ratio, angle of attack and the Reynolds number gave detailed information about the pressure loading, separation condition and the time dependent wake (figure 11). It also showed, rather spectacularly, the effectiveness of the MSBC.

\section{Flat plate and rectangular prisms}

\subsection{Model and wind tunnel test-programme}

Two-dimensional flat plate and rectangular prisms were tested in a $45 \times 45 \mathrm{~cm}$ cross-section wind tunnel with a maximum speed of $50 \mathrm{~m} / \mathrm{s}$. The large converging nozzle at the entrance of the tunnel (contraction ratio $=10: 1$ ) made the flow in the test-section uniform with a level of turbulence less than $0.5 \%$. The tunnel speed was adjusted by a variac transformer and measured using a pitot static tube connected to an inclined alcohol manometer.

A flat plate model, $9 \times 40.5 \mathrm{~cm}$, and two rectangular prism models with a span $(B)$ of $40.5 \mathrm{~cm}$ and depth $(L)$ to width $(H)$ ratio $L / H=0 \cdot 3,1,2,4$ were constructed from Plexiglas. The models were equipped with two moving surface boundary-layer control elements (rotating cylinders) as shown in figure 12. The cylinders were driven by variac controlled AC motors through flexible belt drives. The motor speed was monitored using a strobe light. In the present test-programme the ratio $U c / U$ was varied from 0-3. This corresponded to a maximum cylinder speed of around $11,000 \mathrm{rpm}$ at a free stream speed of $5 \mathrm{~m} / \mathrm{s}$. To ensure two-dimensionality of the flow the models were fitted with end plates. In general, the tunnel speed was kept constant at $5 \mathrm{~m} / \mathrm{s}$, which corresponds to a Reynolds number of $3 \times 10^{4}$ based on the free stream velocity and the model width $(H)$. The lift and drag forces as well as pressure data were recorded over a range of the angle of attack at $5^{\circ}$ increments. The force could be measured with an accuracy of $0.5 \mathrm{gm} / \mathrm{mV}$. Details of the test-arrangement and results are discussed at length in earlier publications (Modi et al 1989, 1990a, 1991b,c).

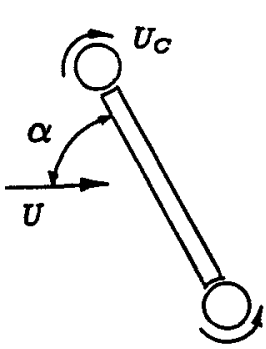

flat plate
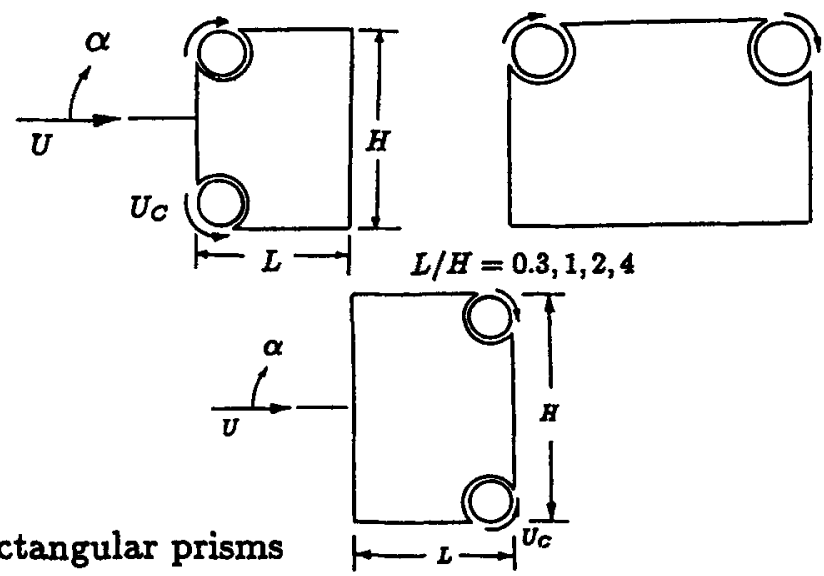

Figure 12. Schematic diagrams of the two-dimensional bluff bodies used during the wind tunnel tests and flow visualization. 


\subsection{Results and discussion}

3.2a Flat plate: Tests with the flat plate were carried out with either of the cylinders rotating independently; or with the two cylinders rotating together, but in the opposite sense, for effective momentum injection to assist in the boundary-layer control. Both the lift as well as the drag results showed remarkable improvement (Modi et al $1990 \mathrm{~b}, \mathrm{c})$.

Of course, the maximum reduction in wake and hence the corresponding decrease in the drag coefficient can be expected when both the cylinders are rotating as shown in figure 13. For $\alpha=90^{\circ}$, a decrease in the drag coefficient from 1.85 at $U c / U=0$ to 0.47 at $U c / U=3$ represents a reduction of around $75 \%$. The flow visualization photographs also showed a remarkable reduction in the wake width thus qualitatively substantiating the trend suggested by the wind tunnel test results.

3.2b Rectangular prisms: Rectangular prisms with rotating cylinders at two adjacent corners provide three basic configurations for study: the side with cylinders facing the flow, forming the top face, or representing the rear face. Various intermediate configurations can be obtained by systematically changing the angle of attack. With four values of $L / H$, to help assess the effect of boundary-layer reattachment and reseparation further downstream, and four values of $U c / U$, the amount of information obtained is rather extensive. Only some typical results in discerning trends are presented here.

Figure 14 shows a sample of the representative results for the experimental phase where the rotating elements are on the top surface, i.e. parallel to the free-stream for $\alpha=0$. Cases corresponding to single- and two-cylinder rotation for the square prism model are considered. At the outset it is apparent that rotation of the second cylinder has very little effect on the flow field, and hence on $C_{D}$, for $\alpha>5^{\circ}$, as now the trailing

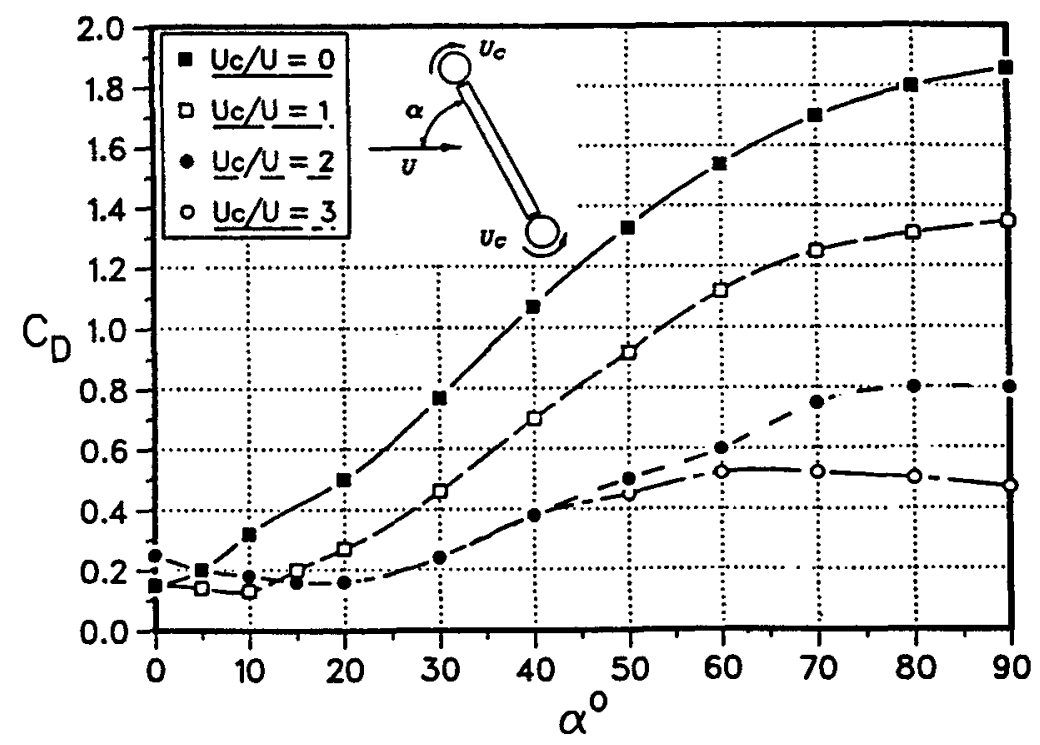

Figure 13. Plots showing significant reduction in drag of a two-dimensional flat plate with the moving surface boundary-layer control applied at both the leading and trailing edges. Note that at $\alpha=90^{\circ}$, the reduction in $C_{D}$ is around $75 \%$. 

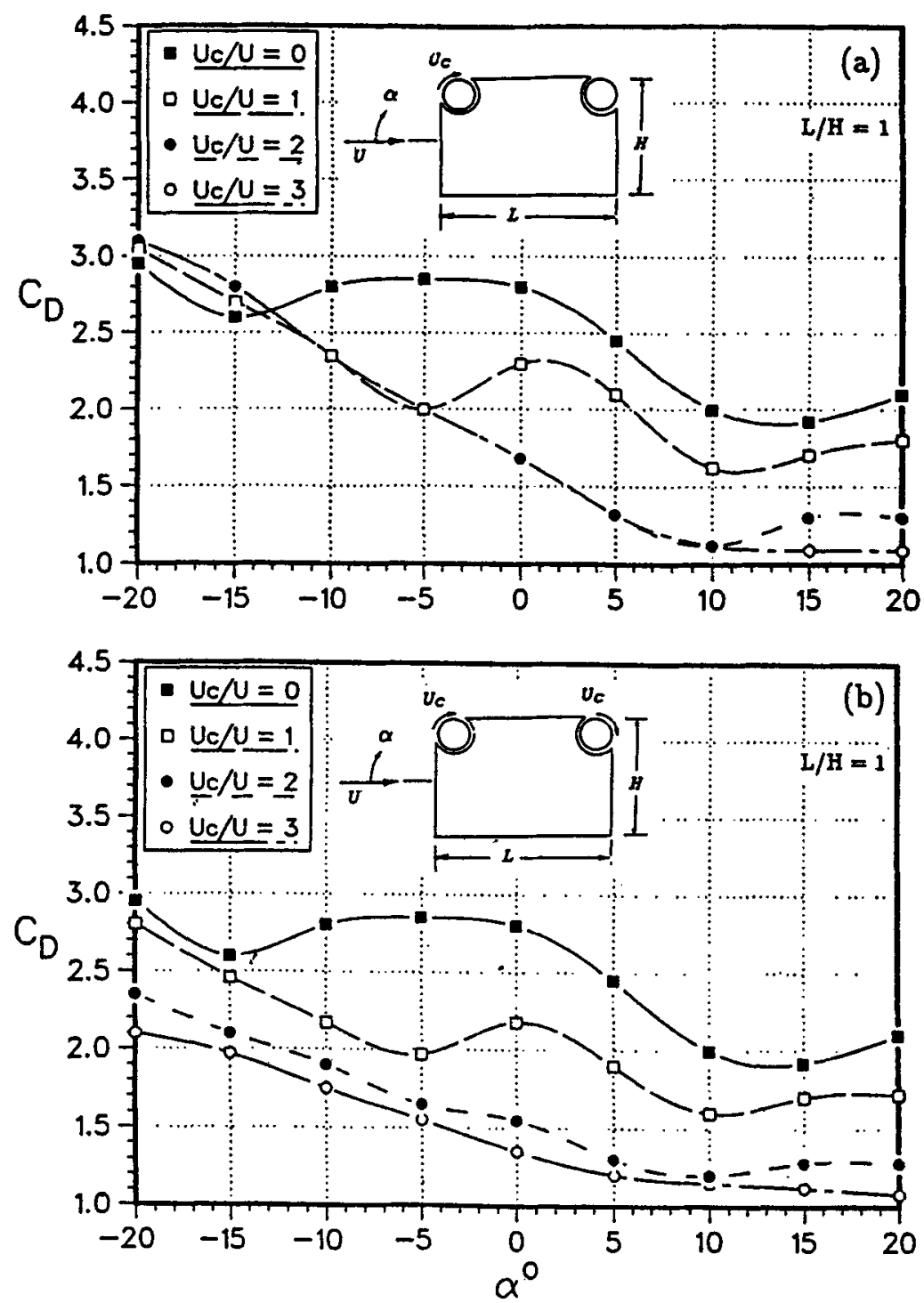

Figure 14. Variation of the drag coefficient with the angle of attack for a twodimensional square prism when the boundary-layer control is applied at the top surface: (a) a rotating cylinder at the leading edge; (b) rotating cylinders at leading and trailing edges.

edge cylinder lies in the wake. However, for smaller and negative $\alpha$ it is quite successful in further reducing $C_{b}$ : from 1.7 at $\alpha=0^{\circ}$ and $U c / U=3$ for the upstream cylinder rotation to 1.3 when both the cylinders are rotating. A reduction in the drag coefficient by $54 \%$ with both the cylinders rotating is indeed quite impressive.

The influence of rotating cylinders located on the rear vertical face of the square and rectangular prisms was also investigated. In this case, the boundary-layer separates at the top and bottom leading edges and the rotating cylinders are 
submerged in the wake thus reducing their effectiveness. Now the reduction in $C_{D} a t$ $\alpha=0^{\circ}$ and $U c / U=3$ was found to be only $13 \%$ for the square prism and virtually zero for the rectangular prism compared to 53 and $40 \%$, respectively, for the case with cylinders at the front face.

\section{Tractor-trailer truck configuration}

\subsection{Background}

A comprehensive literature review of the road vehicle aerodynamics suggests that although aerodynamically contoured car design has become standard practice lately, trucks and buses have changed little during the past 30 years (Sovaran et al 1978; Kramer \& Gerhardt 1980). Most of the modifications have been limited to rounded edges with provision for vanes, skirts and flow deflectors. The benefit due to some of the "add-on" devices is still a matter of controversy and, at best, marginal under conditions other than the specific ones used in their designs. Bearman (1980) has presented an excellent review on the subject (with 54 references cited). The thesis by Wacker (1985) also discusses the limited influence of "add-on" devices with a possibility of increasing the drag under non-optimal conditions. On the other hand, it was found that judicious choice of ground clearance, gap-size between the tractor and trailer, and back inclination can reduce the drag coefficient by a significant amount.

A word concerning numerical analysis of the complex aerodynamics associated with road vehicles would be appropriate. A reliable and cost-effective methodology, if available, can assist in design with reduced dependence on time-consuming and expensive wind tunnel tests. With the advent of supercomputers, parallel processing and neural network concepts, considerable progress has been made in that direction. However, modelling of three-dimensional boundary layers around a complex geometry at supercritical Reynolds numbers, with separation, reattachment and reseparation of unsteady turbulent flows still represents a challenging problem (Kataoka et al 1991).

\subsection{Model and test-procedure}

A 1/12 scale tractor-trailer truck model was constructed out of Plexiglas. The model has a trailer with width $B=22.7 \mathrm{~cm}$, height $H=26.2 \mathrm{~cm}$, length $L=128.4 \mathrm{~cm}$, and a hydraulic diameter of $0.311 \mathrm{~m}$. A typical truck model was supported by four steel guy wires which were suspended from the ceiling and carried turnbuckles to help level the model. As the length of the wire $(\approx 145 \mathrm{~cm})$ is much larger than the maximum horizontal displacement of the truck model $(\leqslant 5 \mathrm{~cm})$, the drag induced displacement was essentially linear in the downstream direction.

Variation in the drag, due to the boundary-layer control devices being relatively small, required development of a sensitive transducer for its measurement. The drag-induced downstream motion of the model was transmitted by an inelastic string to a cantilever beam with a pair of strain gauges near its root. The gauges formed a part of the Wheatstone Bridge (of the Bridge Amplifier Meter, BAM) and the amplified filtered output was recorded using a DISA voltmeter. The sensitivity of the drag measurements was around $0.4 \mathrm{~g} / \mathrm{mV}$. 


\subsection{Results and discussion}

Tests with a scale model of the truck were carried out in the boundary-layer tunnel with negligible blockage effect (blockage ratio $=1.2 \%$ ). The trailer was provided with rotating cylinders at its top leading edge and downstream locations. The $L / H$ ratio for the trailer was approximately 3.75 which suggested that rotation of the rear cylinder has virtually no effect on the drag reduction. The wind tunnel tests substantiated this observation. Considering the fact that:

(i) around $70 \%$ of goods in North America are transported by trucks;

(ii) depending upon the speed, approximately $40-70 \%$ of the power is expended in overcoming the aerodynamic drag;

(iii) on an average, a truck travels around $150,000 \mathrm{~km} /$ year;

even $1 \%$ reduction in the drag coefficient can translate into substantial savings in fuel costs.

With the positive influence of the cylinder roughness on the momentum injection process and associated reduction in drag, it seemed logical to introduce the momentum more directly. This was achieved in several ways:

(i) provide increased cylinder surface roughness through roughness squares, helical grooves or splines running parallel to the cylinder axis;

(ii) keep one cylinder at the top leading edge of the trailer (referred to as the front cylinder) and locate the second cylinder (rear cylinder) at an optimum distance downstream. The objective is to inject additional momentum in the boundarylayer to compensate for dissipation of the momentum introduced by the front cylinder and thus counter the emergence of adverse pressure gradient;

(iii) raise the cylinders so as to immerse them in the boundary layer and assess the effect of cylinder orientation.

Extensive wind tunnel tests with different combinations of speed ratio $(U c / U)$, cylinder location and surface roughness showed the helical groove and spline geometry, with one cylinder located at the leading edge and the other $25.4 \mathrm{~cm}$ downstream, to

Table 1. Wind tunnel tests conducted with different speed ratios and orientation of the twin helical groove and spline cylinders. The front cylinder is located at the top leading edge of the trailer. The second cylinder is located $25.4 \mathrm{~cm}$ (10 in) downstream.

\begin{tabular}{lcc}
\hline & \multicolumn{2}{c}{ Cylinder location } \\
\cline { 2 - 3 } Case & Front raised (mm) & Rear raised (mm) \\
\hline 1 & - & - \\
2 & - & 6.35 \\
3 & - & 12.7 \\
4 & 6.35 & 12.7 \\
5 & 6.35 & - \\
6 & 12.7 & - \\
7 & 12.7 & 6.35 \\
8 & 12.7 & 12.7 \\
9 & 6.35 & 6.35 \\
\hline
\end{tabular}



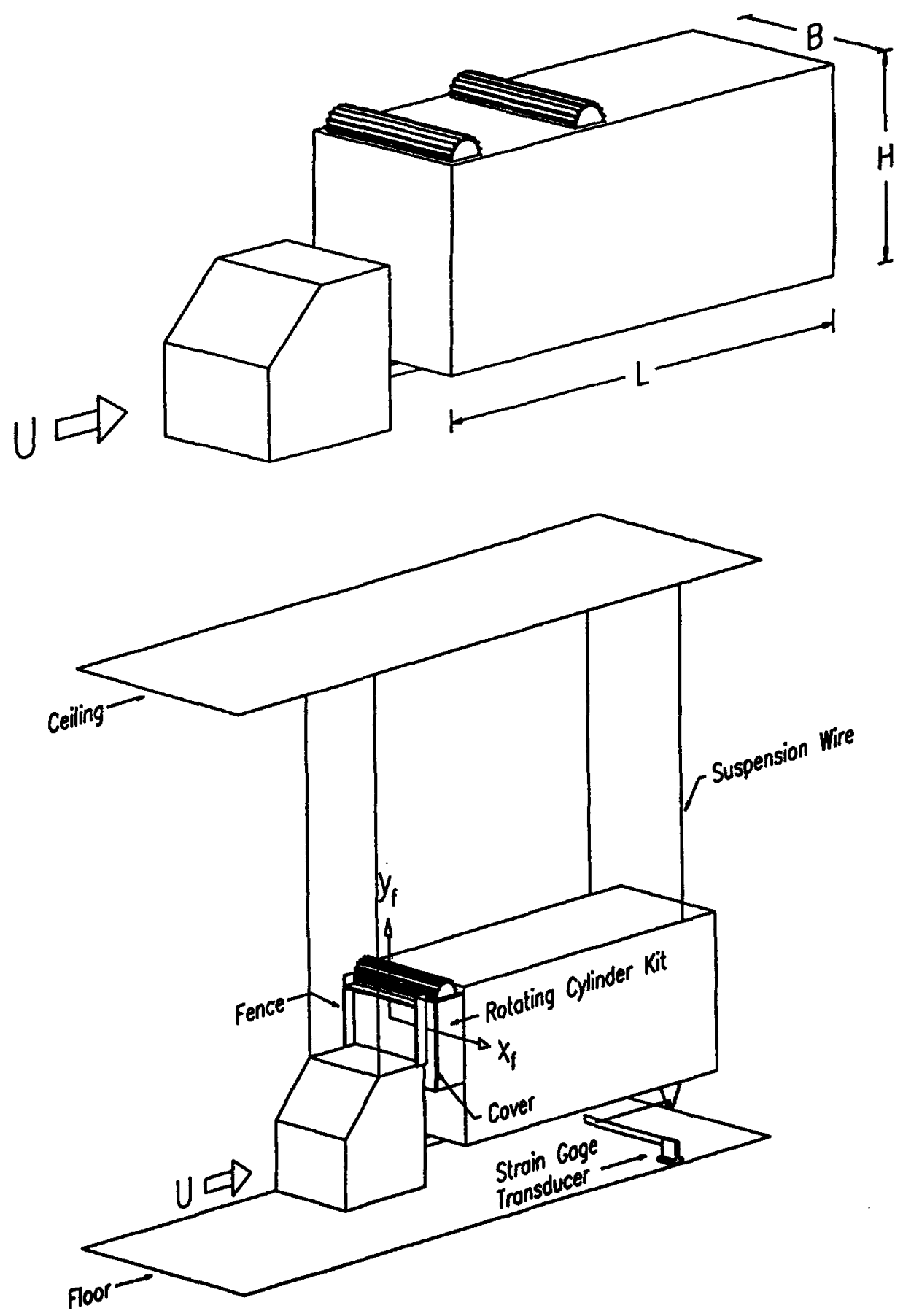

Figure 15. A schematic diagram of the 3-D tractor-trailer truck model and its test-arrangement in the UBC's boundary-layer wind tunnel. 

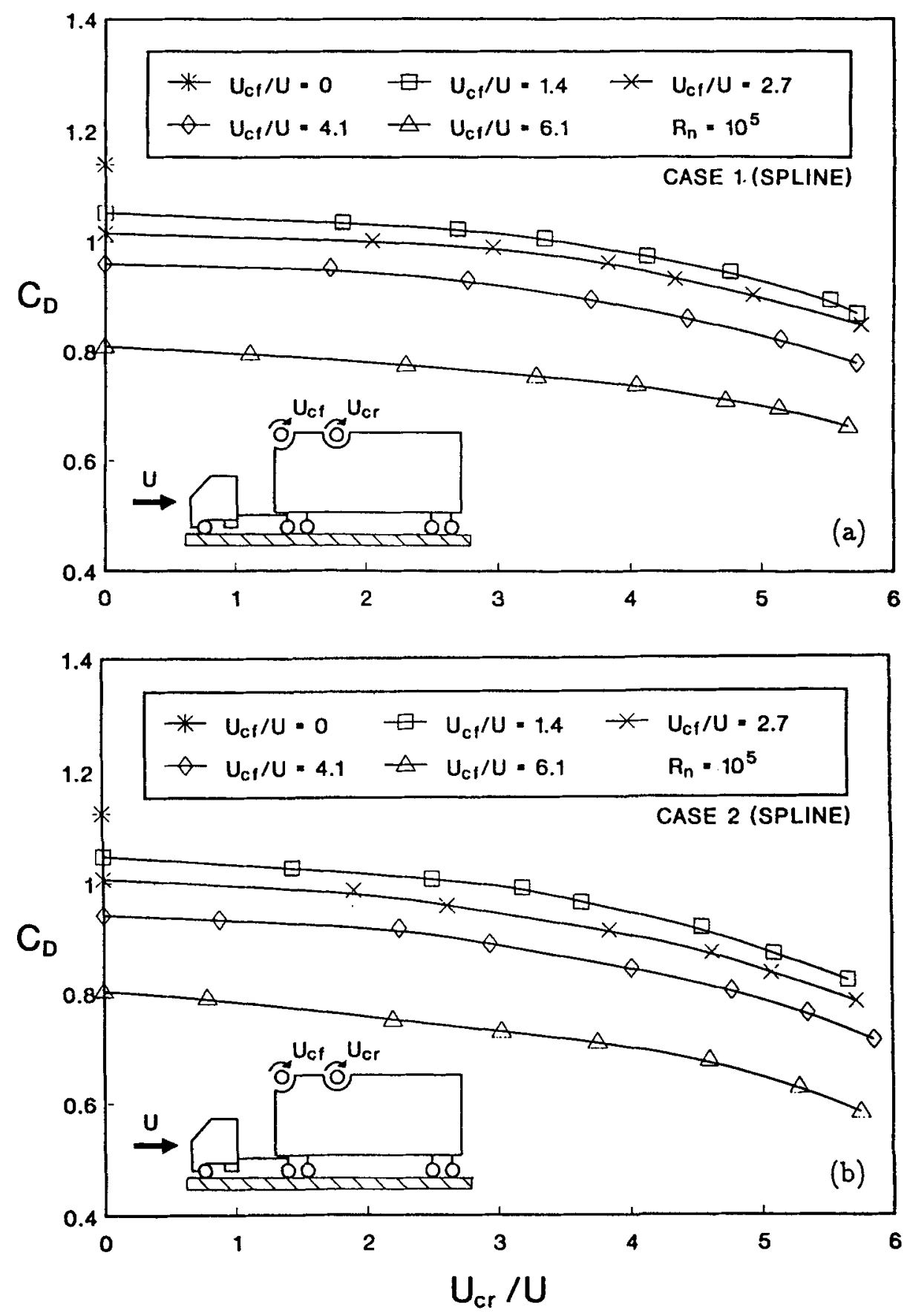

Figure 16. Variation of the drag coefficient $C_{D}$ with the speed ratio for the spline twin-cylinder configuration: (a) Case 1: both cylinders flush; (b) Case 2: front cylinder flush, rear cylinder raised $6.35 \mathrm{~mm}$. 
be quite effective. The effect of raising the cylinder above the trailer surface was also found to be significant. The cylinder orientations studied with helical and spline roughnesses are indicated in table 1 . Figure 15 schematically shows the model and the test arrangement. The extensive amount of information obtained has been reported elsewhere (Modi et al 1991c, pp. 465-82; Ying 1991). Only some typical results are presented here to indicate its potential.

Figure 16a shows the effect of the spline cylinder rotation for case 1, i.e. when both the cylinders are flush with the top face of the trailer. $U_{\mathrm{cf}}$ and $U_{\mathrm{cr}}$ refer to front and rear cylinder surface speeds, respectively. At the outset it is apparent that the front cylinder rotation $\left(U_{\mathrm{cr}}=0\right)$ reduces the drag coefficient rather significantly, from $1 \cdot 14$ at $U_{\mathrm{cf}}=0$ to 0.96 at $U_{\mathrm{cf}}=4.1$, a drop of around $15.8 \%$. Rotation of the rear cylinder improves the situation further and for both the cylinders with a speed ratio $U c / U=4 \cdot 1$, the reduction in $C_{D}$ reaches $22.8 \%$.

The effect of raising the rear cylinder is shown in figure $16 \mathrm{~b}$. Note that even in absence of the momentum injection $\left(U_{\mathrm{cf}}=U_{\mathrm{cr}}=0\right)$, the reference drag coefficient is slightly reduced $\left(C_{D}=1 \cdot 19\right)$. This may be attributed to the combined effect of an increase in the projected area on which the drag coefficient is based and the large wake width caused by the rear cylinder. Rotation of the front cylinder does not seem to improve the situation significantly (compared to case 1 ), as for $U_{\text {cf }} / U=4 \cdot 1$, the reduction in drag is $16.8 \%$. With both the cylinders rotating at a speed ratio of $4 \cdot 1$, the decrease in drag coefficient amounts to $24.8 \%$.

Essentially the same trend continued to persist when the front cylinder was also raised (case 7). The drag coefficient in absence of the cylinder rotation dropped further to $1 \cdot 12$ as explained before. With $U_{\mathrm{cf}} / U=U_{\mathrm{cr}} / U=4 \cdot 1$, the reduction in drag reached almost $26 \%$. Thus the splined geometry of the rotating elements with raised positions, appears quite promising in reducing the pressure drag of the tractor-trailer truck configuration through MSBC.

\section{Control of wind-induced instabilities}

With the success of the moving surface boundary-layer control, in increasing lift and reducing drag of both slender bodies at high angles of attack and bluff geometries, attention was directed towards control of wind induced instabilities.

The response of aerodynamically bluff bodies when exposed to fluid streams has been a subject of considerable study for quite some time. The prevention of aeroelastic vibrations of smokestacks, transmission lines, suspension bridges, tall buildings etc. is of particular interest to engineers. Ever since the pioneering contribution by Strouhal, who correlated periodicity of the vortex shedding with the diameter of a circular cylinder and the velocity of the fluid stream, there has been a continuous flow of important contributions resulting in a vast body of literature. This has been reviewed rather adequately by Cermak (1975), Modi \& Slater (1991), Welt (1988) and others. In general, the oscillations may be induced by vortex resonance or geometricfluid dynamic instability called galloping.

Several passive devices such as helical strakes, shrouds, slats, tuned mass and nutation dampers etc. have been proposed over the years (figure 17) and have exhibited varying degrees of success in minimizing the effects of vortex induced and galloping types of instabilities (Zdravkovich 1980). In general, vibration suppressing devices tend to change the aerodynamic characteristics of the structure in such a way as to 
(a)

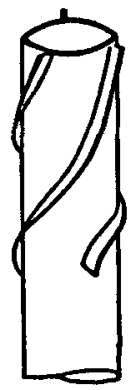

Helical Strakes
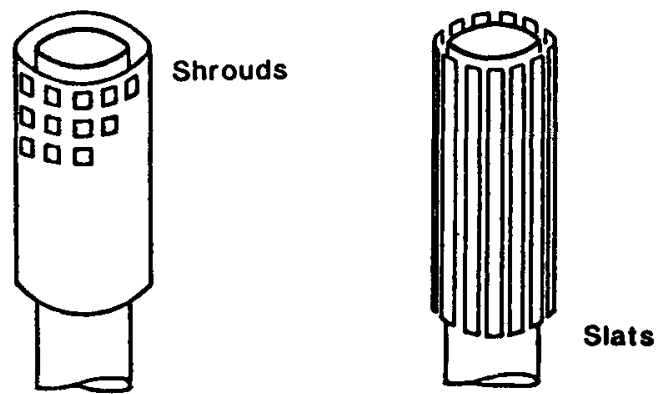

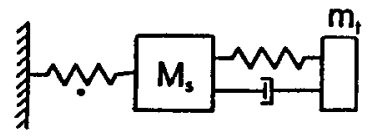

Tuned Mass Damper

(b)

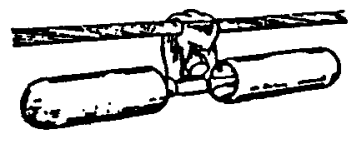

Stockbridge Damper

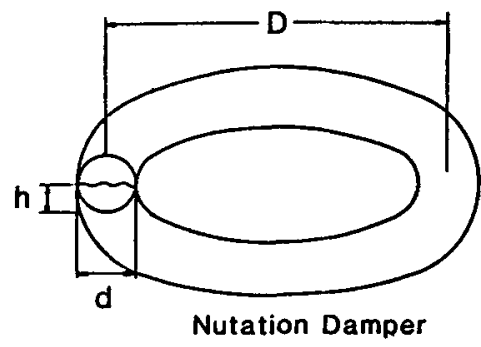

Figure 17. Passive devices used to control wind induced instabilities: (a) strakes, slats, and shrouds modify system aerodynamics: (b) dampers provide energy dissipation mechanism.

interfere with and weaken the existing force; while the dampers provide a mechanism for dissipating energy. It is of interest to note that all the above mentioned procedures are passive in character. Semi-active devices such as a rotating element for the boundary-layer control and, through it, damping of the instability has received virtually no attention. Such applications of the MSBC were explored only recently (Kubo et al 1991; Modi et al 1991d).

Variation of the Strouhal number (S) with Reynolds number (Re) for the flat plate with rounded tips due to the presence of cylinders but in the absence of their rotation is shown in figure 18a. As expected, at a given Reynolds number, $\mathrm{S}$ diminishes as the angle of attack increases. Effect of the cylinder rotation on the Strouhal number as a function of $\alpha$ for a fixed $\operatorname{Re}$ of $3 \times 10^{4}$ is presented in figure 18b. The classical results of Fage \& Johansen (1927) are also included for comparison. A remarkable increase in the Strouhal number with cylinder rotation at a given $\alpha$ is apparent. It suggests an increase in the shedding frequency corresponding to narrowing of the wake. Obviously, this will delay the onset of vortex resonance.

Kubo et al (1991) applied the concept to a two-dimensional square prism provided with twin rotating elements at the corners of the front face. The tests were carried out in a wind tunnel with the spring supported model free to undergo plunging oscillations. The single cylinder rotation affected the coherence of the vortex shedding and hence suppressed resonant instability. On the other hand, cylinder rotation successfully modified the loading to arrest the galloping. Excellent flow visualization pictures supported the wind tunnel test observations.

After an uncertain beginning and interrupted advances over nine decades, it is 

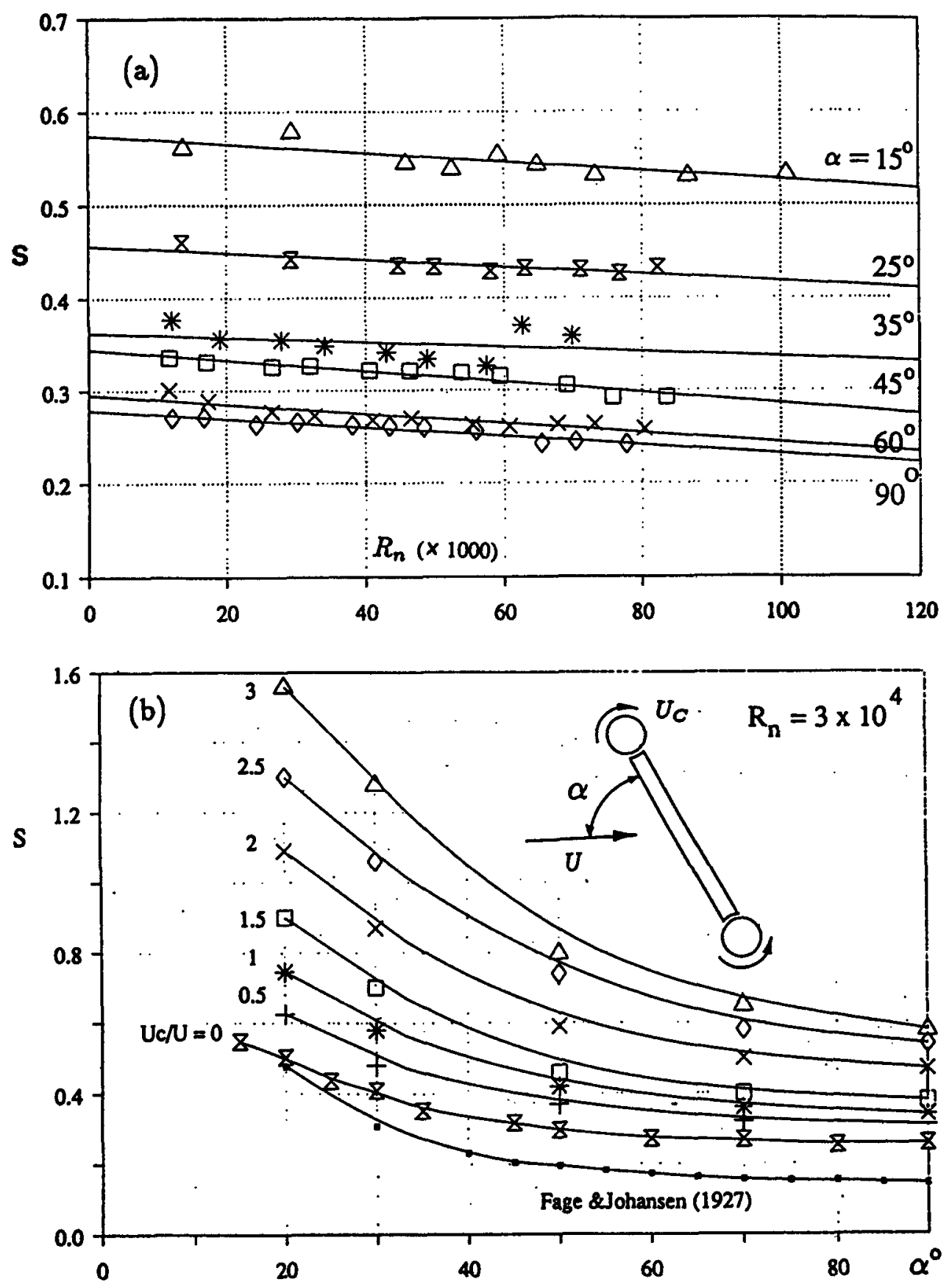

Figure 18. Strouhal number (S) associated with a flat plate having rounded edges due to tip cylinders: (a) variation with Reynolds number and angle of attack in the absence of cylinder rotation; (b) effect of momentum injection. 
apparent that the field now presents an exciting opportunity for contributions. As the Gita puts it:

"Knowledge is merely a small island surrounded by a vast ocean of ignorance"

No matter how far we advance, we will always be on the shores of that uncharted ocean. But then, a journey fulfills itself at every step.

\section{Concluding remarks}

Based on a rather fundamental study of moving surface boundary-layer control with two-dimensional airfoils, plates and prisms, as well as its application to a scale model of a typical tractor-trailer truck configuration, the following conclusions can be made:

(i) Moving surface boundary-layer control (MSBC) can significantly increase lift, decrease drag and delay stall of an aircraft. Its application to the next generation of high performance airplanes is indeed quite exciting. NASA and the US Air Force are actively looking into this aspect;

(ii) the concept also appears to be quite promising in reducing the drag of bluff bodies. For the flat plate at $\alpha=90^{\circ}$, it reduced the drag coefficient by $75 \%$. The maximum reduction for a square prism varied from $54 \%(L / H=1)$ to $40 \%$ $(L / H=2)$;

(iii) effectiveness of the momentum injecting device diminishes when located in the wake;

(iv) surface roughness of the rotating cylinder tends to improve the boundary-layer control;

(v) the MSBC concept also proved effective in reducing the drag of a truck configuration by $26 \%$;

(vi) the concept is essentially semi-passive in character requiring a negligible amount of power for its implementation;

(vii) numerical approach to the problem using the surface singularity procedure, as well as finite element and finite difference application to integration of the Navier-Stokes equations, presents considerable scope for contribution.

(viii) flow visualization study confirms the effectiveness of the MSBC quite dramatically.

(ix) application of the concept in arresting wind-induced vortex resonance and galloping instabilities appears quite promising.

The models were fabricated in the Mechanical Engineering Workshop. The assistance of $\mathrm{M} / \mathrm{s}$ E Abell, $\mathrm{P}$ Hurren and D Camp in the design and construction of the models is gratefully acknowledged. The investigation was supported by the Natural Sciences and Engineering Research Council of Canada, Grant No. A-2181.

\section{References}

Bearman PW 1980 Review of bluff body flows applicable to vehicle aerodynamics. Trans. ASME, J. Fluids Eng. 102: 265-274

Betz A 1961 History of boundary layer control in Germany. Boundary layer and flow control (ed.) G V Lachmann (London: Pergamon) vol. 1 
Cebeci T, Bradshaw P 1977 Momentum transfer in boundary layer (Washington: HemisphereMcGraw Hill)

Cebeci T, Smith A M O 1974 Analysis of turbulent boundary layers (New York: Academic Press)

Cermak J E 1975 Application of fluid mechanics to wind engineering: Freeman Scholar Lecture. Trans. ASME, J. Fluids Eng. 97: 9-38

Chang P K 1970 Separation of flow (New York: Pergamon)

Fage A, Johansen F C 1927 On the flow of air behind and inclined flat plate of infinite span. Proc. R. Soc. London A116: 170-197

Favre A 1938 Contribution a L'etude Experimentale des Mouvement Hydrodynamiques a Deux Dimensions, doctoral thesis presented to the University of Paris

Flettner A 1925 The Flettner rotor ship. Engineering 19: 117-120

Goldstein S 1938 Modern developments in fluid mechanics (Oxford: University Press) vols. 1 \& 2

Iverson J D 1972 Correlation of magnus force data for slender spinning cylinders. $A I A A$ 2nd Atmospheric Flight Mechanics Conference, Palo Alto, California, Paper No. 72-966

Kataoka T, China H, Nakagawa K, Yanagimoto K, Yoshida M 1991 Numerical simulation of road vehicle aerodynamics and effect of aerodynamic devices. SAE International Congress and Exposition, Detroit, Paper No. 91-0597

Keller H B, Cebeci T 1972 Accurate numerical methods for boundary-layer flows, Part 2. Two-dimensional turbulent flow. AIAA J. 10: 1193

Kramer C, Gerhardt H J 1980 Road vehicle aerodynamics. Proceedings of the 4th Colloquium on Industrial Aerodynamics (Aachen, Germany: Fachhochschule)

Kubo Y, Modi V J, Yasuda H, Kato K 1991 On the suppression of aerodynamic instabilities through the moving surface boundary-layer control. Proceedings of the 8th International Conference on Wind Engineering (ed.) A G Davenport (London, Ont: University of Westen Ontario)

Lachmann G V 1961 Boundary layer and flow control (London: Pergamon) vols. $1 \& 2$

Magnus G 1853 Ueber die Verdichtung der Gase an der Oberflache Glatter Korper. Poggendorfs Ann. Phy. Chem. 88: 604-610

Modi V J 1991 Moving surface boundary-layer control: Experiments, analysis and applications. The G I Taylor Memorial Lecture 36th ISTAM Congress (Bombay: Indian Inst. Technol India

Modi V J, Fernando M S U K, Yokomizo T 1989 Moving surface boundary layer control as applied to two- and three-dimensional bodies. Proceedings of the 8th Colloquium on Industrial Aerodynamics-Industrial Flows (eds.) C Kramer, H Gerhardt (Aachen, Germany: Fachhochschule) pp. 73-84

Modi V J, Fernando M S U K, Yokomizo T 1990a Drag reduction of bluff bodies through moving surface boundary layer control. 28th Aerospace Sciences Meeting, Reno, Nevada, Paper No. AIAA-90-0298

Modi V J, Fernando M S U K, Yokomizo T 1991a Moving surface boundary-layer control: Studies with bluff bodies and application. AIAA J. 29: 1400-1406.

Modi V J, Fernando M S U K, Yokomizo T 1991b Moving surface boundary-layer control as applied to two and three dimensional bodies. J. Wind Eng. Ind. Aerodyn. 38: 83-92

Modi V J, Mokhtarian F 1985 Joukowsky airfoil with circulation control. AIAA 12th Atmospheric Flight Mechanics Conference, Snowmass, Colorado, Paper No. 85-1772-CP

Modi V J, Mokhtarian F, Yokomizo T 1988 Effect of moving surfaces on the airfoil boundarylayer control. Proc. AIAA Atmospheric Flight Mechanics Conference (ed.) J E Benek (New York: AIAA) paper. no. AIAA-88-4337

Modi V J, Mokhtarian F, Yokomizo T, Ohta G, Oinuma T 1987a Bound vortex boundarylayer control with application to V/STOL airplanes. Proceedings of the IUTAM Symposium on Fundamental Aspects of Vortex Motion Tokyo, Japan, pp. 196-199

Modi V J, Mokhtarian F, Yokomizo T, Ohta G, Oinuma T 1987b Vortex motion (eds) H Hasimoto, T Kambe (Amsterdam: North-Holland)

Modi V J, Slater J E 1991 Unsteady aerodynamics and vortex induced aeroelastic instability of a structural angle section. Proceedings of the 13th Biennial ASME Conference on Mechanical Vibration and Noise (eds) T C Huang et al (New York: ASME) DE-vol, 37, pp. 177-188

Modi V J, Swinton P G, McMillan K, Lake P, Mullins D, Akutsu T 1979 On the boundary layer control using the concept of moving surface. Proceedings of CANCAM 79 (eds) F. Ellyin, K W Neale, vol. 2, pp. 621-622 
Modi V J, Swinton P G, McMillan K, Lake P, Mullins D, Akutsu T 1980 Moving surface boundary layer control for aircraft operation at high incidence. Proceedings of the AIAA 7th Atmospheric Flight Mechanics Conference, Danvers, MA, Paper No. 80-1621, pp. 515-522

Modi V J, Swinton P G, McMillan K, Lake P, Mullins D, Akutsu T 1981 J. Aircraft 18: 963-968

Modi V J, Ying B, Yokomizo T 1990b Boundary-layer control of bluff bodies through momentum injection. SAE International Bus/Truck Meeting and Exposition Detroit, Paper No. 90-2225

Modi V J, Ying B, Yokomizo T 1990c SAE Trans., J. Commercial Vehicles 99: 778-794

Modi V J, Ying B, Yokomizo T 1991c An approach to design of the next generation of fuel efficient trucks through aerodynamic drag reduction. Proceedings of the ASME Winter Annual Meeting (eds) S A Velinsky, R M Fries, I Haque, D Wang (New York: ASME) DE-vol. 40, pp. $465-482$

Modi V J, Ying B, Yokomizo T 1991d Effect of momentum injection on the aerodynamics of several bluff bodies. Proceedings of the 8th International Conference on Wind Engineering, (ed.) A G Davenport (in press)

Modi V J, Yokomizo T 1992 On the boundary-layer control through momentum injection: Numerical flow visualization and experimental studies. Proceedings of the 6th International Symposium on Flow Visualization (eds) Y Tanida, H Miyashiro (Berlin: Springer-Verlag)

Mokhtarian F 1988 Fluid dynamics of airfoils with moving surface boundary-layer control. Ph D thesis, University of British Columbia, Vancouver

Mokhtarian F, Modi V J 1984 Fluid dynamics of airfoils with circulation control for V/STOL application. Proc. AIAA 11th Atmospheric Flight Mechanics Conference (ed.) L B Schiff (New York: AIAA) paper no. AIAA-84-2090

Mokhtarian F, Modi V J 1986 Fluid dynamics of airfoils with moving surface boundary-layer control. Proc. AIAA Atmospheric Flight Mechanics Conference (New York: AIAA) paper no. AIAA-86-2184 CP

Mokhtarian F, Modi V J 1988 Fluid dynamics of airfoils with moving surface boundary layer control. J. Aircraft 25: 163-169

Mokhtarian F, Modi V J, Yokomizo T 1988 Rotating air scoop as airfoil boundary-layer control. J. Aircraft 25: 973-975

National Research Council 1966 Report of the Research Co-ordination Group on Boundary Layer Control to Suppress Separation, Associate Committee on Aerodynamics

Rosenhead L 1966 Laminar boundary layers (Oxford: University Press)

Schlichting H 1968 Boundary-layer theory (New York: McGraw-Hill)

Sovaran G, Morel T, Mason T W Jr 1978 Aerodynamic drag mechanisms of bluff bodies and road vehicles. Proceedings of the Symposium held at the General Motors Research Laboratories (New York: Plenum)

Swanson W M 1961 The magnus effect: A summary of investigation to date. Trans. ASME, Basic Eng. 83: 461-470

Thwaites B 1960 Incompressible aerodynamics (London: Clarendon)

Wacker T 1985 A preliminary study of configuration effects on the drag of a tractor-trailer combination. MASc thesis, University of British Columbia, Vancouver

Welt $\mathrm{E} 1988 \mathrm{~A}$ study of nutation dampers with application to wind-induced oscillations, $\mathrm{Ph} \mathrm{D}$ thesis, University of British Columbia, Vancouver

Ying B 1991 Boundary-layer control of bluff bodies with application to drag reduction of tractortrailer truck configurations. MASc thesis, University of British Columbia, Vancouver

Zdravkovich M M 1980 Review and assessment of effectiveness of various aero and hydromechanic means for suppressing vortex shedding. Proceedings of the 4th Colloquium on Industrial Aerodynamics (eds) C Kramer et al (Aachen, Germany: Fachhochschule) part 2, pp. $29-46$ 